\title{
P03-5-22 Poster session
}

\section{DHA supplementation prevent the progression of NASH via GPR120 signaling}

Kazuo Nakamoto ${ }^{1}$, Akira Hirasawa ${ }^{2}$, Shogo Tokuyama ${ }^{1}$

${ }^{I}$ Department of Clinical Pharmacy, Kobe Gakuin University, Japan, ${ }^{2}$ Department of Genomic Drug Discovery Science, Kyoto University, Japan

Nonalcoholic steatohepatitis (NASH) is one of the most common liver diseases involving chronic accumulation of fat and inflammation, often leading to advanced fibrosis, cirrhosis and carcinoma. However, the pathological mechanism for this is unknown. GPR120/FFAR4 has been recognized as a functional fatty acid receptor and an attractive therapeutic target for metabolic diseases. In this study, we investigated the involvement of GPR120/FFAR4 in the pathogenesis of NASH. Mice fed with a $0.1 \%$ methionine and choline deficient high-fat (CDAHF) diet showed a significant increase in plasma aspartate transaminase and alanine transaminase levels, fatty deposition, inflammatory cell infiltration, and mild fibrosis. Docosahexaenoic acid (DHA, a GPR120/FFAR4 agonist) suppressed the inflammatory cytokines in the liver tissues and prevented fibrosis in the wild type (WT) mice fed CDAHF diet, but not GPR120/FFAR4 deficient (GPR120KO) mice. GPR120KO mice fed CDAHF diet showed increment of the number of crown like structures and the immunoreactivity for F4/80 positive cells, and increased TNF- $\alpha$ mRNA in the liver compared to WT mice fed CDAHF diet. GPR120 KO mice fed CDAHF diet showed more severe liver inflammation than that of WT mice fed CDAHF diet, but not fibrosis. Our findings suggest that DHA supplementation could be prevented the development of NASH via GPR120/FFAR4 signaling. Furthermore, decrease of GPR120/FFAR4 signaling could be facilitated an inflammatory response in the process of NASH progression. (Eur J Pharmacol., in press) 